\title{
Complexity of a matter-field Hamiltonian in the vicinity of a quantum instability
}

\author{
A.M. Kowalski ${ }^{\mathrm{a}, \mathrm{b}, *}$, A. Plastino ${ }^{\mathrm{a}, \mathrm{c}}$, R. Rossignoli ${ }^{\mathrm{a}, \mathrm{b}}$ \\ a Instituto de Física (IFLP-CCT-Conicet), Fac. de Ciencias Exactas, Universidad Nacional de La Plata, C.C. 727,1900 La Plata, Argentina \\ ${ }^{\mathrm{b}}$ Comision de Investigaciones Científicas (CIC), Argentina \\ ' Argentina's National Research Council (CONICET), Argentina
}

\section{H I G H L I G H T S}

- We study the very complex dynamics of a non-linear Hamiltonian describing the interaction between a quantum system and a classical one.

- This dynamics is relevant for quantum optics and condensed matter.

- Ours is a statistical description of the pertinent dynamical features.

- These features include chaos, unstability, unboundedness, periodicity, and quasi-periodicity.

- Statistical analysis provides a better understanding of these features than a mere dynamic portrayal of them.

\section{A R T I C L E I N F O}

\section{Article history:}

Received 5 May 2018

Received in revised form 17 May 2018

Available online 17 August 2018

\section{Keywords:}

Statistical description versus dynamical one

Complexity

Entropy

Semiquantum system

Poincare sections

Time series

Bandt-Pompe's probabilities extraction

\section{A B S T R A C T}

Using as information-quantifiers the entropy and the statistical complexity, we analyze the rich, complex dynamics of a special non linear Hamiltonian $H$. $H$ describes the interaction between a quantum system and a classical one. The concomitant system exhibits periodicity, quasi-periodicity, not-boundedness, and chaotic regimes. The chaotic phenomenon, together with complex dynamics, arise in the vicinity of an unstable case, that of the purely quantum system.

(C) 2018 Elsevier B.V. All rights reserved.

\section{Introduction}

In the last 70 years [1], statistical quantifiers based on information theory (see for instance [2-4], and references therein) have become quite useful for dynamics' characterization purposes, where the dynamics emerges from a time series.

Bandt and Pompe (BP) [5,6] advanced a symbolic formalism for assessing the probability distribution (PD) $P$ linked to an arbitrary time series (see Appendix). Their approach is based on peculiar facets of the attractor-rebuilding problem via causal information, that is duly incorporated into the process of building up the pertinent PD. A remarkable BP-result is a notable performance-improvement in what concerns the information quantifiers one finds in using their PD-generating technique. One only needs to assume that the system is stationary and that enough data are available.

\footnotetext{
* Corresponding author at: Instituto de Física (IFLP-CCT-Conicet), Fac. de Ciencias Exactas, Universidad Nacional de La Plata, C.C. 727, 1900 La Plata, Argentina.

E-mail addresses: kowalski@fisica.unlp.edu.ar (A.M. Kowalski), plastino@fisica.unlp.edu.ar (A. Plastino), rossigno@fisica.unllp.edu.ar (R. Rossignoli).
} 
Now, a topic of great interest is that of the interplay between quantum and classical systems. If quantum effects in one of the systems are small vis-a-vis those of the other, regarding it as classical not only simplifies the description but provides profound insight into the composite system's dynamics. One may cite as illustrations the Bloch equations [7], twolevel systems interacting with an electromagnetic field within a cavity, the Jaynes-Cummings semi-classical model $[8,9]$, collective nuclear features [10], etc.

We are interested in symbolically analyzing the classical-quantum interplay in the bipartite system described above. This is of interest in both Quantum Optics and Condensed Matter [8,9,11,12], particularly in view of the fact that we deal with a bosonic system that admits quasi-periodic and unbounded regimes, separated by an unstable region [13]. This feature makes the interaction with a classical mode a quite attractive phenomenon.

Our all important PDs are extracted from times series à la BP, while the time series are obtained from the Poincare-sections arising from a non linear system of equations, that represents the extant dynamics.

Section 2 deals with our semi-quantum system's dynamics. In particular, Section 2.1 gives results for the isolated quantum system while Section 2.2 does so for the composite system. In Section 3 we exhaustively analyze our information quantifiers while Section 4 displays the pertinent results. Finally, some conclusions are drawn in Section 5.

\section{The semiquantum model}

Focus attention upon the Hamiltonian [14]

$$
H=\varepsilon_{+}\left(b_{+}^{\dagger} b_{+}+\frac{1}{2}\right)+\varepsilon_{-}\left(b_{-}^{\dagger} b_{-}+\frac{1}{2}\right)+(\Delta+\alpha X)\left(b_{+} b_{-}+b_{-}^{\dagger} b_{+}^{\dagger}\right)+\frac{\omega}{2}\left(P_{X}^{2}+X^{2}\right),
$$

where $b_{ \pm}^{\dagger}, b_{ \pm}$are boson creation and annihilation operators satisfying the standard commutation relations $\left(\left[b_{\mu}, b_{v}^{\dagger}\right]=\delta_{\mu \nu}\right.$, $\left[b_{\mu}, b_{v}\right]=\left[b_{\mu}^{\dagger}, b_{v}^{\dagger}\right]=0$ for $\left.\mu, v= \pm\right)$, while $\varepsilon_{ \pm}>0$ are the single boson energies, and $X, P_{X}$ represent classical coordinate and momentum quantities, with $\omega$ the associated oscillator's frequency.

The quantum dynamical equations are the canonical ones [11,12], that is, operators $O$ evolve in the Heisenberg picture as

$$
i \frac{d O}{d t}=-[H, O] .
$$

The pertinent evolution equation for the mean value $\langle 0\rangle \equiv \operatorname{Tr}[\rho O(t)]$ becomes

$$
i \frac{d\langle O\rangle}{d t}=-\langle[H, O]\rangle,
$$

with the average being taken with respect to a proper quantum density matrix $\rho$. Moreover, classical variables obey the classical Hamilton's equations of motion

$$
\begin{aligned}
\frac{d X}{d t} & =\frac{\partial\langle H\rangle}{\partial P_{X}}, \\
\frac{d P_{X}}{d t} & =-\frac{\partial\langle H\rangle}{\partial X} .
\end{aligned}
$$

The set of Eqs. $(3)+(4)$ is an autonomous one of coupled, first-order ordinary differential equations (ODE), that permit a dynamical description such that no quantum rule is violated. Particularly, commutation-relations are trivially timeconserved, since the quantum evolution is the canonical one for our effective time-dependent Hamiltonian. Note that $X$ can be viewed as a time-dependent parameter of our quantal system. The initial conditions are determined by the quantum density matrix $\rho$. Pass now to the hermitic operators $N=b_{+}^{\dagger} b_{+}+b_{-}^{\dagger} b_{-}, \delta N=b_{+}^{\dagger} b_{+}-b_{-}^{\dagger} b_{-}, O_{+}=b_{+} b_{-}+b_{-}^{\dagger} b_{+}^{\dagger}$, $O_{-}=i\left(b_{+} b_{-}-b_{-}^{\dagger} b_{+}^{\dagger}\right)$, and we are able to recast our Hamiltonian (1) as

$$
H=\varepsilon(N+1)+\gamma \delta N+(\Delta+\alpha X) O_{+}+\frac{\omega}{2}\left(P_{X}^{2}+X^{2}\right),
$$

where $\varepsilon=\left(\varepsilon_{+}+\varepsilon_{-}\right) / 2>0$ and $\gamma=\left(\varepsilon_{+}-\varepsilon_{-}\right) / 2$, with $|\gamma|<\varepsilon$. From Eqs. (3)-(4) we thus encounter a closed system of equations for our set of quantum mean values plus classical variables:

$$
\begin{aligned}
\frac{d\langle N+1\rangle}{d t} & =2(\Delta+\alpha X)\left\langle O_{-}\right\rangle, \\
\frac{d\left\langle O_{-}\right\rangle}{d t} & =2(\Delta+\alpha X)\langle N+1\rangle+2 \varepsilon\left\langle O_{+}\right\rangle, \\
\frac{d\left\langle O_{+}\right\rangle}{d t} & =-2 \varepsilon\left\langle O_{-}\right\rangle, \\
\frac{d X}{d t} & =\omega P_{X}, \\
\frac{d P_{X}}{d t} & =-\left(\omega X+\alpha\left\langle O_{+}\right\rangle\right),
\end{aligned}
$$


where $d\langle\delta N\rangle / d t=0$.

Eqs. (6) are clearly a nonlinear ODEs set. Non-linearity has been inserted by the coupling between the two systems, governed by the parameter $\alpha$. For $\alpha=0$ the two systems become decoupled, of course, and the precedent equations become, as a consequence, those for two independent linear systems.

The expectation value $\left\langle O_{-}\right\rangle$is regarded as a "current", while $\left\langle O_{+}\right\rangle$yields the mean value of the quantum component of the interaction potential. Each level population is fixed by $\left\langle b_{ \pm}^{\dagger} b_{ \pm}\right\rangle=(\langle N\rangle \pm\langle\delta N\rangle) / 2$. The full system (6) displays moreover the Bloch-like motion-invariant

$$
I=\langle N+1\rangle^{2}-4\left|\left\langle b_{+} b_{-}\right\rangle\right|^{2}=\langle N+1\rangle^{2}-\left\langle O_{-}\right\rangle^{2}-\left\langle O_{+}\right\rangle^{2},
$$

that fulfills $d I / d t=0$ in both the linear $(\alpha=0)$ and nonlinear $(\alpha \neq 0)$ instances, as it is easily seen.

Given that $\langle\delta N\rangle$ is conserved, it makes sense to work with the effective energy $E_{\text {eff }}=\langle H\rangle-\gamma\langle\delta N\rangle-\varepsilon$ in place of the total energy $\langle H\rangle$. The two quantities are motion-invariants. Employing I together with $E_{\text {eff, }}$ we diminish the amount of freedom-degrees of the system (6) to just three, which enables the employment of important tools like the Poincare sections so as to investigate the system's dynamics.

\subsection{Quantum subsystem}

For $\alpha=0$, the quantum system's is fully fixed by the quantum Hamiltonian

$$
H_{q}=\varepsilon_{+}\left(b_{+}^{\dagger} b_{+}+\frac{1}{2}\right)+\varepsilon_{-}\left(b_{-}^{\dagger} b_{-}+\frac{1}{2}\right)+\Delta\left(b_{+} b_{-}+b_{-}^{\dagger} b_{+}^{\dagger}\right) .
$$

The dynamics of this system is analyzed using a method advanced in [13,15], that allows for diagonalization of general quadratic forms, even if they lack positivity. The pertinent dynamics displays three different regimes, according to the relation $\Delta-\varepsilon[13]$.

(I) A stable regime, for $|\Delta|<\varepsilon$, with an evolution that is bounded and quasi-periodic. The system can be separated into two traditional normal modes. This regime can further be divided into three sub-regimes according to the $H-$ spectrum [13]. Always, discreteness and quasi-periodicity prevail (see [13]).

(II) A dynamically unstable one, for $|\Delta|>\varepsilon$. The dynamics is exponentially unbounded. The system can be split up into two normal modes. However, the creation and annihilation operators for them are non-hermitian (see [13]).

(III) A non-separable case for $|\Delta|=\varepsilon$. Here $H$ can no longer be cast as a sum of two-independent modes [13]. We are here at the border between the stable and unstable regimes.

\subsection{Results for our composite system}

The different regimes above are conditioned by the relation amongst $\varepsilon, \Delta$, and $\alpha$, no matter what the initial conditions and $\omega$ 's value are. (I) For $|\alpha| \geq \varepsilon$, the dynamics is always unbounded [14]. (II) For $\varepsilon>|\alpha|$, the dynamics is determined by $\varepsilon, \Delta$ and $\alpha . \varepsilon$ "competes" with the two coupling constants ( $\Delta$ and $\alpha$ ), but as $\alpha$ diminishes, the system tends to the linear scenario and thus the relation between $\Delta$ and $\varepsilon$ becomes dominant. In [14] one observes illustrative Poincare sections (Figs. 2,3 , and 6). For instance, if $\alpha<\varepsilon$ is kept fixed but the ratio $\varepsilon / \Delta$ is varied, it is seen that if $\varepsilon>|\Delta|$ the dynamics is periodic and turns into quasi-periodic in the neighborhood of the non-diagonalizable regime $\varepsilon=|\Delta|$, displaying growing nonlinear artifacts as this region is reached (Fig. 2c of [14]). If $\varepsilon<|\Delta|$ things become unbounded. One notes the same behavior for distinct values of $\alpha<\varepsilon$, if we keep the same ratio $\varepsilon / \Delta$. For augmenting values of $\alpha / \Delta$, one again detects evolution from periodic curves to rather complex, quasi-periodic ones. Finally, one confronts chaos.

The most noticeable behavior takes place at the critical case $\varepsilon \simeq|\Delta|$, in the neighborhood of the non-separable instance of the linear system, at the border with the unbounded region. We encounter complex quasi-periodic evolution curves. Additionally, for appropriate "small" values of $\alpha(\alpha<\Delta)$, chaos is seen to arise.

\section{Entropy and statistical complexity as information measures}

An information measure (IM) is a quantity that suitably characterizes probability distributions (PDs). We are interested in physical processes described by a $\mathrm{PD} P=\left\{p_{j}, j=1, \ldots, N\right\}$, where $N$ is the number of available states of the physical system. In the present work we restrict to the Shannon Entropy and the Statistical Complexity (SC). We consider the normalized Shannon Entropy $\mathcal{H}$ as

$$
\mathcal{H}[P]=\mathrm{S}[P] / \mathrm{S}\left[P_{e}\right],
$$

where

$$
\mathrm{S}[P]=-\sum_{j=1}^{N} p_{j} \ln \left(p_{j}\right),
$$


and $\mathrm{S}\left[P_{e}\right]=\ln N$, the entropy corresponding to the uniform distribution $P_{e}$. As a second IM we will use the product form for the SC advanced in [3]

$$
\mathcal{C}[P]=\mathcal{H}[P] \cdot \mathcal{Q}[P],
$$

where $\mathcal{Q}[P]$ is called the disequilibrium, defined [6] via the Jensen-Shannon divergence $J_{S}[4]$

$$
J_{S}\left[P_{1}, P_{2}\right]=\mathrm{S}\left[\frac{P_{1}+P_{2}}{2}\right]-\mathrm{S}\left[P_{1}\right] / 2-\mathrm{S}\left[P_{2}\right] / 2 .
$$

$J_{S}$ is the symmetric form of the Kullback-Leibler relative entropy. Its square root is a metric [4]. It is a extensive quantity in the thermodynamical sense [4]. Thus, the associated statistical complexity becomes an intensive quantity. We take

$$
\mathcal{Q}[P]=\mathcal{Q}_{0} \cdot \mathcal{J}_{S}\left[P, P_{e}\right],
$$

where $\mathcal{Q}_{0}$ is a normalization constant $(0 \leq \mathcal{Q} \leq 1)$. The maximum disequilibrium obtains when one of the components of $P$, say $p_{k}$, is unity and the remaining components vanish. The disequilibrium $\mathcal{Q}$ reflects on the systems' "structure", becoming different from zero only if there exist "privileged" states among the accessible ones.

Remark that $\mathcal{C}$ is not a trivial function of the entropy. It depend on two different probabilities distributions, namely, (1) one associated to the system under analysis, $P$, and (2) the uniform distribution $P_{e}$. Additionally, it is well known that for a given $\mathcal{H}$ value, a range of possible $\mathrm{SC}$ values exists, from a minimum one $\mathcal{C}_{\min }$ up to a maximal value $\mathcal{C}_{\max } \mathcal{C}$ yields then absolutely new information. A general method to find the bounds $\mathcal{C}_{\min }$ and $\mathcal{C}_{\max }$ associated to the generalized $\mathcal{C}=\mathcal{H} \cdot \mathcal{Q}$-quantities can be looked up in Ref. [16]. It is obvious that relevant information regarding the correlational structures amongst the components of a physical system can be gained from the statistical complexity.

As a way of verifying our results we will use also a second, alternative definition of disequilibrium, given by the following form for $\mathcal{Q}_{S}[P][2]$

$$
\mathcal{Q}_{S}[P]=1-\mathcal{H}[P] .
$$

Next Section numerically analyzes our system's dynamics via the two quantifiers just described.

\section{Results}

The initial conditions used here are consistent with an appropriate density operator, so that the uncertainty relationships of the quantum system remain satisfied for all times. The resulting accuracy of our calculations was checked by verification of the time-constancy of the dynamical invariants $E_{\text {eff }}$ and $I$ (within a $10^{-10}$ precision).

Time series (TS) to construct our PDs $P$ are obtained from the systems' Poincare sections (PS). An alternative procedure is to get the PDs from phase space's curves, and we have done so as well. Obviously, PS's are better representatives of phase space than particular curves in it. The numerical results we obtained verify this assertion.

Our PD's are extracted from the TS via the Bandt-Pompe approach (see Appendix). The succession of PS's used in our calculations are obtained via crossings with a plane: solutions of (6) with the $X(t)=0$ plane. This is done for identical values of the invariants $E_{\text {eff }}$ and $I$. One also varies $\varepsilon / \Delta$ and fixes both $\alpha / \Delta$ and $\omega / \Delta$ for the whole PS's succession.

For each PS associated to a given $\varepsilon / \Delta>1$ we consider 21 curves, generated by varying the initial conditions $\left\langle O_{-}\right\rangle_{0}$ and $P_{0}$ (maintaining compatibility with the chosen values for $E_{\text {eff }}$ and $I$ ). In the considered unbounded zone $(\varepsilon / \Delta=1)$ we need 10000 curves. $X_{0},\langle N\rangle_{0}$, and $\left\langle O_{+}\right\rangle_{0}$ are kept constant. Moreover, for each PS our TS is that corresponding to values, as a function of time, of different quantities like $\langle N+\rangle,\left\langle O_{-}\right\rangle,\left\langle O_{+}\right\rangle$, etc. The plots displayed here correspond to the $\left\langle O_{+}\right\rangle$-case. One obtains similar results for either of these quantities. We took, per PS, 10000 crossing-points with the plane $X(t)=0$. We can ascertain that both entropy and statistical complexity statistically represent, in appropriate fashion, the results yielded by pure dynamical tools. More to the point, these two quantities allow for a more schematic and general characterization of our results. This assessment of the value of out two quantifiers constitutes a most valuable point.

Fig. 1 displays $\mathcal{H}$ vs. $\varepsilon / \Delta$. Decreasing $\varepsilon / \Delta, \mathcal{H}$ grows (with slight oscillations) from the quasi-periodic zone $(\varepsilon / \Delta>1.2)$ towards $\varepsilon / \Delta=1$, till becoming maximal at $\varepsilon / \Delta \simeq 1$.05. Chaoticity suddenly emerges therein [14]. Afterwards, $\mathcal{H}$ suddenly drops in the unbounded dynamics' zone $(\varepsilon / \Delta \simeq 1)$ till reaching an absolute minimum at $(\varepsilon / \Delta=1)$.

Important $\mathcal{H}$-variations are appreciated in the region between $\varepsilon / \Delta \simeq 1.2$ and $\varepsilon / \Delta \simeq 1.05$, corresponding to the entropic maximum. Dynamically, this regions is associated to a zone in which non-linearity become of a more involved nature. This happens as we reach $\varepsilon / \Delta=1.05$, in the vicinity of $\varepsilon / \Delta=1$, value corresponding to the quantum unstable scenario. Remember that here we cannot attain separability into quantum normal modes.

Fig. 2 displays the statistical complexity (SC) vs. $\varepsilon / \Delta$. Roughly, $\mathcal{C}$ behaves like $\mathcal{H}$. See that if $\varepsilon / \Delta$ decreases, SC grows till $\varepsilon / \Delta \simeq 1.2$. Onwards, it strongly oscillates till $\varepsilon / \Delta \simeq 1.08$, reaching an absolute maximum. Herefrom, $\mathcal{C}$ suddenly diminishes, reaching an absolute minimum in the unbounded zone.

Even if the minima are attained at the same $\varepsilon / \Delta$-value, the maxima of $\mathcal{H}$ and $\mathcal{C}$ are not attained in the same way. SC reaches its maximum sooner than entropy in the process of approaching the unstable point (quantal). These maxima-differences in $\varepsilon / \Delta$ might be due to numerical artifacts. However, Figs. 3 and 4 allow for a different interpretation. 


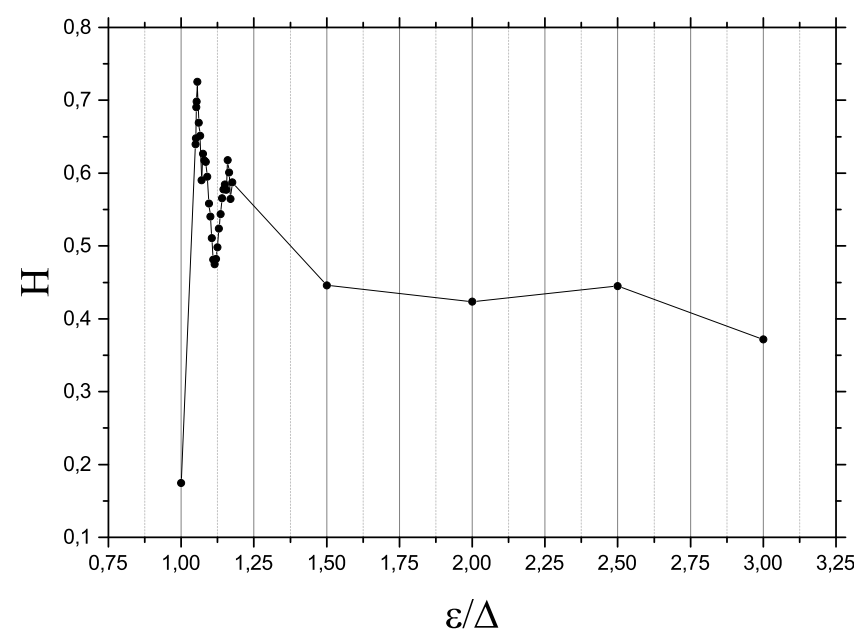

Fig. 1. Entropy $\mathcal{H}$ vs. $\varepsilon / \Delta$. $\mathcal{H}$ is calculated with PDFs extracted from Poincare sections for the $X=0$ plane, corresponding to $E_{\text {eff }}=4.8$ and $I=4$, with $X_{0}=1,\langle N\rangle_{0}=1$ and $\left\langle O_{+}\right\rangle_{0}=0$. We set $\omega / \Delta=1$ and $\alpha / \Delta=0.015$ while the ratios $\varepsilon / \Delta$ are varying. Decreasing $\varepsilon / \Delta, \mathcal{H}$ grows (with oscillations) from the quasi-periodic zone $(\varepsilon / \Delta>1.2)$ towards $\varepsilon / \Delta=1$, till becoming maximal at $\varepsilon / \Delta \simeq 1.05$. Chaos suddenly emerges therein. Afterwards, $\mathcal{H}$ suddenly drops in the unbounded dynamics' zone $(\varepsilon / \Delta \simeq 1)$ till reaching an absolute minimum at $(\varepsilon / \Delta=1)$.

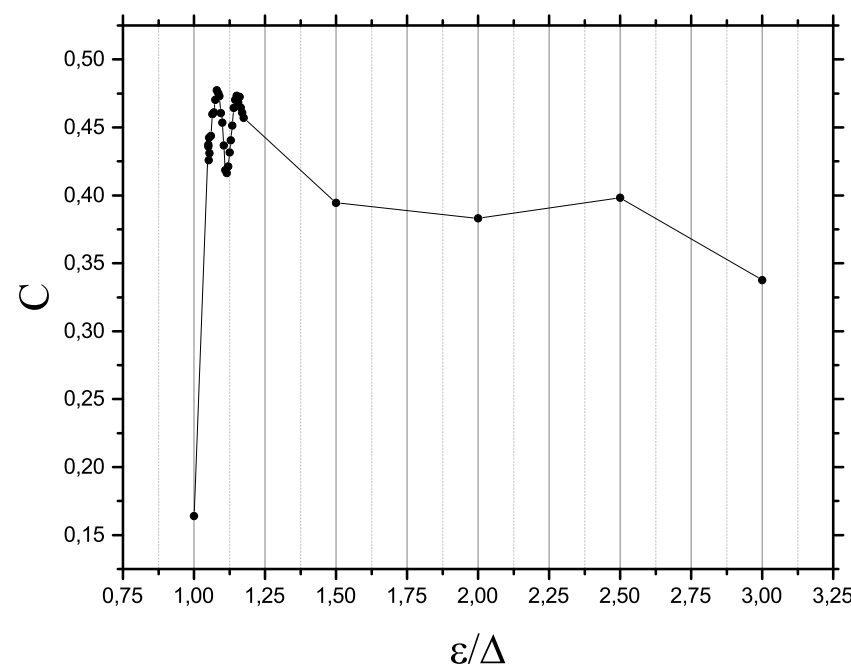

Fig. 2. Statistical Complexity $\mathcal{C}$ vs. $\varepsilon / \Delta$, calculated from the same Poincare Sections as in Fig. 1. Roughly, $\mathcal{C}$ behaves like $\mathcal{H}$. See that if $\varepsilon / \Delta$ decreases, $\mathcal{C}$ grows till $\varepsilon / \Delta \simeq 1$. Onwards, it strongly oscillates till $\varepsilon / \Delta \simeq 1.08$, reaching an absolute maximum. Herefrom, $\mathcal{C}$ suddenly diminishes, reaching an absolute minimum in the unbounded zone.

Fig. 3 depicts the disequilibrium $\mathcal{Q}$ vs. $\varepsilon / \Delta$. There is a $\mathcal{Q}$-absolute maximum at the value for which there is an absolute entropic minimum, and vice versa, as one should expect for consistency's sake. At the value $\varepsilon / \Delta \simeq 1.08$, where there is a complexity-maximum, $\mathcal{Q}$ reaches a local minimum (indicated in the figure as $\varepsilon / \Delta_{\mathrm{CMax}}$ ). $\mathcal{H}$ monotonously decreases in the vicinity of this point. All these results seem to provide us with a rather coherent picture.

Fig. 4 plots $\mathcal{C}$ vs. $\mathcal{H}$. It is clear that $C$ is not a function of $H$, as indicated in Section 3. Further, we see that while these two quantities reach their minima at the same $\varepsilon / \Delta$, this is not so for their maxima. It can be seen that the maximum of $\mathcal{C}$ occurs at the $\mathcal{H}$-value indicated by $H_{C M a x}$. It seems dynamically reasonable that the complexity should attain its maximum before $\mathcal{H}$ does so, in the process of approaching the vicinity of the unstable quantal point above referred to, just before chaos ensues.

Fig. 5 displays the second version of disequilibrium (D) $\mathcal{Q}_{S}$, given by Eq. (14) together with the complexity $\mathcal{C}_{S}$, as evaluated with $\mathcal{Q}_{s}$. We use it as a check of the correctness of the previous results. Our new graphs are coherent with what is depicted in Figs. 2 and 3. Absolute maxima and minima for the two disequilibriums are attained for, respectively, $\varepsilon / \Delta=1$ and $\varepsilon / \Delta \simeq 1.05$. The specifical values are, of course, different in the two D-cases. The pertinent plotting-scales are also different (see Fig. 5a). 


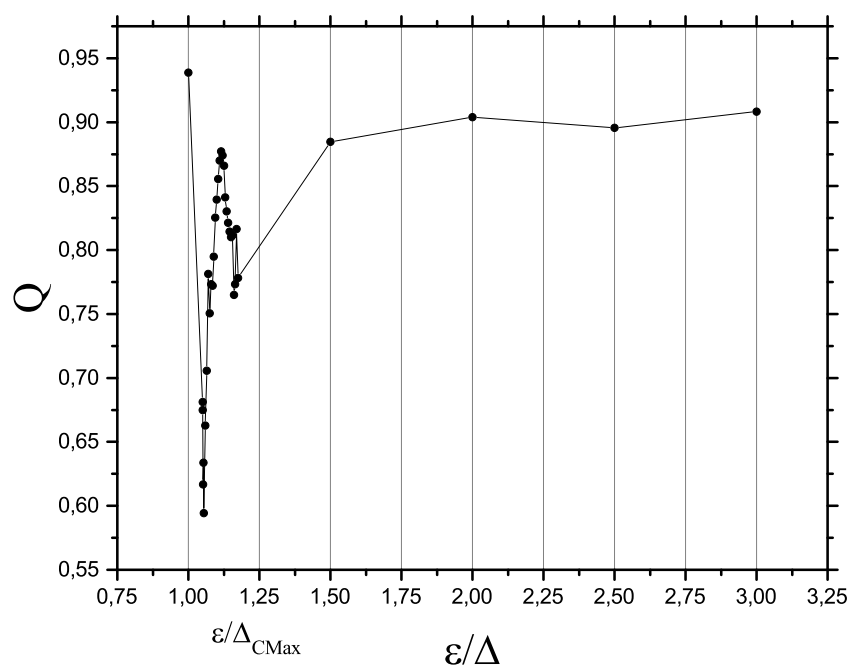

Fig. 3. Disequilibrium $\mathcal{Q}$ vs. $\varepsilon / \Delta$, calculated from the same Poincare Sections as in Fig. 1 . There is a $\mathcal{Q}$ - absolute maximum at the value for which there is an absolute entropic minimum, and vice versa. At the value $\varepsilon / \Delta \simeq 1.08$, where there is a complexity-maximum, $\mathcal{Q}$ reaches a local minimum (indicated as $\left.\varepsilon / \Delta_{\text {CMax }}\right) . \mathcal{H}$ monotonously decreases in the vicinity of this point. All these results seem to provide us with a rather coherent picture.

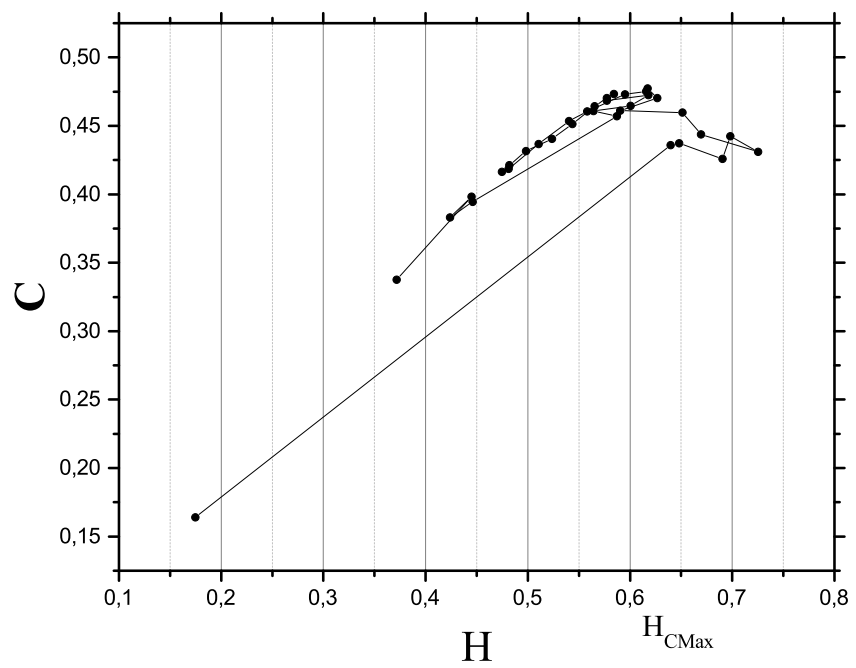

Fig. 4. We plot $\mathcal{C}$ vs. $\mathcal{H}$. Minima of the two quantities take place at the same $\varepsilon / \Delta$ but maxima do not. It can be seen that the maximum of $\mathcal{C}$ occurs at the $\mathcal{H}$-value indicated by $H_{\text {CMax }}$.

The absolute maximum of the second complexity-version $\mathcal{C}_{S}$ appears at $\varepsilon / \Delta=1.125$ (Fig. $5 \mathrm{~b}$ ), close but different to $\varepsilon / \Delta=0.8$ obtained for $\mathcal{C}$. Further, we see a more noticeable drop in $\mathcal{C}$ for $\varepsilon / \Delta>1.2$. The main different detail obtained using the second $\mathrm{D}$ is that of the maximum complexity-location.

\section{Conclusions}

We have analyzed, using statistical tools, a peculiar dynamical problem: that of a non lineal hamiltonian $H$. $H$ describes the interaction of a quantum system with a classical field, being of interest in quantum optics and in condensed matter $[8,9,11,12]$. The isolated quantum system is a bosonic one that admits of both unbounded and quasi-periodic regimes. These two regimes are separated by an unstable third one [13]. In turn, the classical field is represented by a single-mode electromagnetic one.

The ensuing, a very rich dynamics of the composite system is ruled by a system of ordinary differential equations (ODE), given in (6). It displays periodic, quasi-periodic, unbounded, chaotic, and non-linear sub-dynamics, depending on the $H$ parameters' value. Interestingly is that both the (1) complex non-linear and (2) chaotic sub-dynamic lie, in the parameters' 

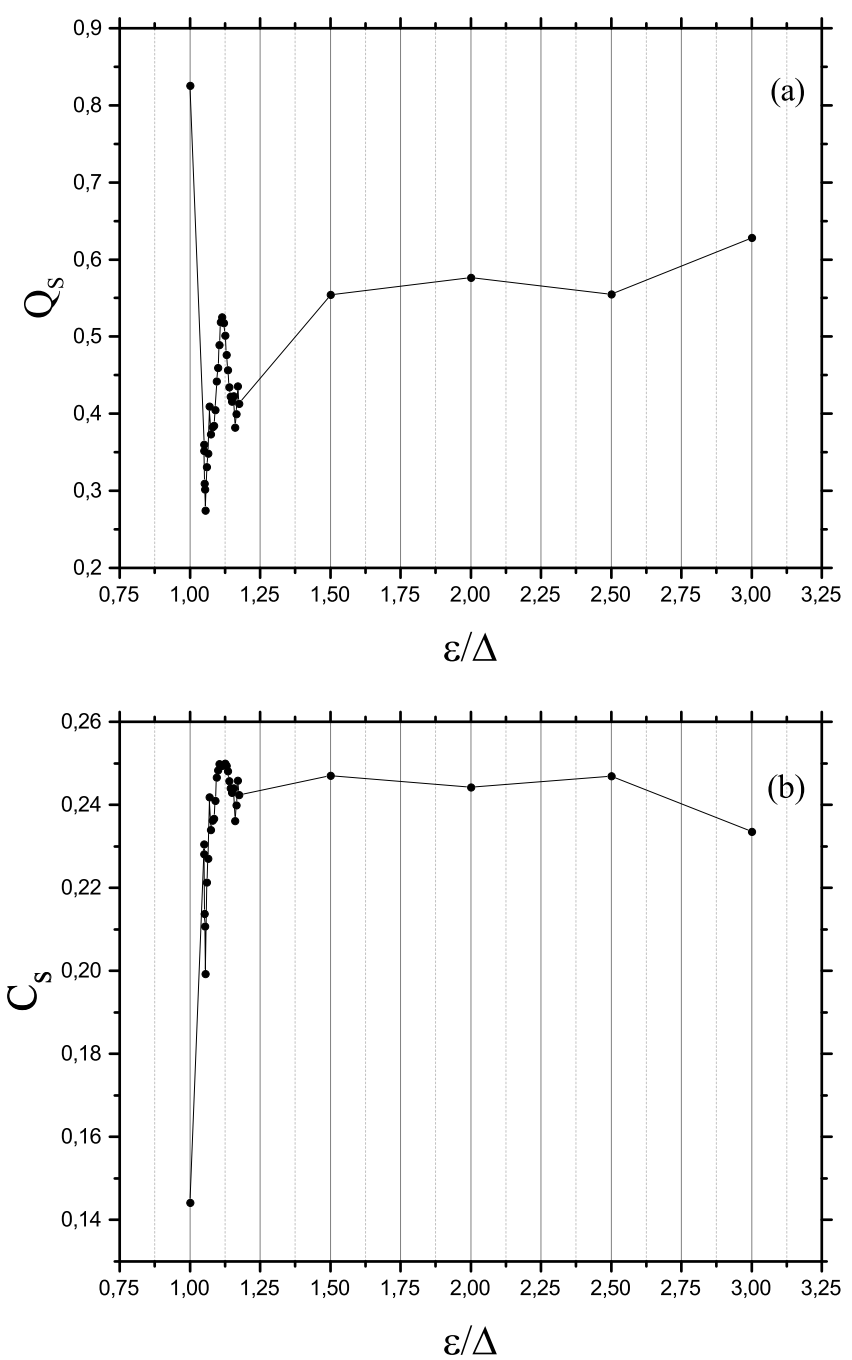

Fig. 5. Fig. 5a display $\mathcal{Q}_{S}$ vs. $\varepsilon / \Delta$ (see Eq. (14). Fig. 5b. shows $\mathcal{C}_{S}$ calculated with $\mathcal{Q}_{S}$ vs. $\varepsilon / \Delta$. These graphs are coherent with what is depicted in Figs. 2 and 3. Absolute maxima and minima for the two disequilibriums are attained for, respectively, $\varepsilon / \Delta=1$ and $\varepsilon / \Delta \simeq 1.05$. The specifical values are, of course, different in the two D-cases. The pertinent plotting-scales are also different (Fig. 5a). The absolute maximum of the second complexity-version $\mathcal{C}_{S}$ appears at $\varepsilon / \Delta=1.125$ (Fig. $5 \mathrm{~b}$ ), close but different to $\varepsilon / \Delta=0.8$ obtained for $\mathcal{C}$.

space, in the vicinity of the unstable (purely) quantum one. Although the presence of the classical system enables the existence of non-linearity and chaos, one can reasonably assume, then, that (1) and (2) emerge from the quantum system.

Our statistical tools are the entropy and the statistical complexity, evaluated via symbolic analysis from time-series (TS). This is a result of evaluating relevant probability distributions using the Bandt-Pompe technique. In turn, the TS were obtained from Poincare sections (PS) derived via our ODE system. We get the PS, through intersections of the ODE's solutions of (6) with the $X(t)=0$ plane, keeping constant the invariants $E_{\text {eff }}$ and $I$. In our graphs we also keep constant (i) the values of $\alpha / \Delta$ and $\omega / \Delta$ and (ii) the initial conditions $X_{0},\langle N\rangle_{0}$ and $\left\langle O_{+}\right\rangle_{0}$ (for all the PS-succession). One varies $\varepsilon / \Delta$.

We guarantee that both entropy and statistical complexity are statistical representatives of the results obtained by purely dynamical tools. Our two statistical quantifiers yield a more schematic and general description of the system than the dynamical results. We emphasize the absolute entropy maximum (EM) at the chaotic zone and the absolute complexity maximum taking place just before (in parametric space) the EM occurs, that is, as $\varepsilon / \Delta$ diminishes in its way to the unbounded region, all of this happening near the unstable quantum zone. Remark that we are here providing a statistical picture practically unobtainable via pure dynamical reasoning.

\section{Acknowledgments}

AK and RR acknowledge support from CIC of Argentina. AP acknowledges support from CONICET of Argentina. 


\section{Appendix A. PD Based on Bandt and Pompe's methodology}

To use the Bandt and Pompe [5] methodology for evaluating the probability distribution $P$ associated with the time series (dynamical system), one starts by considering partitions of the pertinent $D$-dimensional space that will hopefully "reveal" relevant details of the ordinal structure of a given one-dimensional time series $\mathcal{S}(t)=\left\{x_{t} ; t=1, \ldots, M\right\}$, with embedding dimension $D>1$ and time delay $\tau$. We will take here $\tau=1$ as the time delay, a parameter of the approach [5]. We are interested in "ordinal patterns", of order $D$ [5,17], generated by

$$
(s) \mapsto\left(x_{s-(D-1)}, x_{s-(D-2)}, \ldots, x_{s-1}, x_{s}\right),
$$

which assigns to each time the $D$-dimensional vector of values at times $s, s-1, \ldots, s-(D-1)$. Clearly, the greater the $D$-value, the more information on the past is incorporated into our vectors. By "ordinal pattern" related to the time $(s)$, we mean the permutation $\pi=\left(r_{0}, r_{1}, \ldots, r_{D-1}\right)$ of $[0,1, \ldots, D-1]$ defined by

$$
x_{S-r_{D-1}} \leq x_{S-r_{D-2}} \leq \cdots \leq x_{S-r_{1}} \leq x_{S-r_{0}} .
$$

In this way the vector defined by Eq. (A.1) is converted into a unique symbol $\hat{x}_{i}$. Thus, a permutation probability distribution $P_{x}=\left\{p\left(\hat{x}_{i}\right), i=1, \ldots, D !\right\}$ is obtained from the time series $x_{i}$. The probability distribution $P$ is obtained once we fix the embedding dimension $D$ and the time delay $\tau$. The former parameter plays an important role for the evaluation of the appropriate probability distribution, since $D$ determines the number of accessible states, $D$ !, and tells us about the necessary length $M$ of the time series needed in order to work with a reliable statistics. The whole enterprise works for $D$ ! $\ll M$. In particular, Bandt and Pompe [5] suggest for practical purposes to work with $3 \leq D \leq 7$. For more details see [17]. We have considered in this work $D=6$, a reasonable value given in the literature for series of length $M=10000$. We have checked the results taking $D=5$, obtaining similar descriptions for the information measures considered.

\section{References}

[1] C.E. Shannon, Bell Syst. Technol. J. 27 (1948) 379; 623.

[2] J.S. Shiner, M. Davison, P.T. Landsberg, Phys. Rev. E 59 (1999) 1459.

[3] R. López-Ruiz, H.L. Mancini, X. Calbet, Phys. Lett. A 209 (1995) 321.

[4] P.W. Lamberti, M.T. Martin, A. Plastino, O.A. Rosso, Physica A 334 (2004) 119

[5] C. Bandt, B. Pompe, Phys. Rev. Lett. 88 (2002) 174102.

[6] A.M. Kowalski, M.T. Martín, A. Plastino, O.A. Rosso, Physica D 233 (2007) 21.

[7] E. Bloch, Phys. Rev. 70 (1946) 460.

[8] P. Milonni, M. Shih, J.R. Ackerhalt, Chaos in Laser-Matter Interactions, World Scientific Publishing Co., Singapore, 1987.

[9] P. Meystre, M. Sargent, Elements of Quantum Optics, Springer, NY, 1991.

[10] P. Ring, P. Schuck, The Nuclear Many-Body Problem, Springer-Verlag, Berlin, Germany, 1980

[11] A.M. Kowalski, A. Plastino, A.N. Proto, Phys. Rev. E 52 (1995) 165.

[12] A.M. Kowalski, Physica A 458 (2016) 106.

[13] R. Rossignoli, A.M. Kowalski, Phys. Rev. A 72 (2005) 032101.

[14] A.M. Kowalski, R. Rossignoli, Chaos Solitons Fractals 109 (2018) 140.

[15] R. Rossignoli, A.M. Kowalski, Phys. Rev. A 79 (2009) 062103.

[16] M.T. Martín, A. Plastino, O.A. Rosso, Physica A 369 (2006) 439

[17] M. Zanin, L. Zunino, O.A. Rosso, D. Papo, Entropy 14 (2012) 1553. 